\title{
Primitive Neuroectodermal Tumor Arising From the Kidney
}

\author{
Jack Wells, $M D$
}

Primitive neuroectodermal tumor is a type of sarcoma that occurs in the first two decades of life. ${ }^{1}$ It usually appears as a soft-tissue mass in the chest wall and paraspinal region. This tumor has been said to be the soft-tissue equivalent of the Ewing sarcoma and malignant small cell tumor of the thoracopulmonary region. ${ }^{2}$ It usually arises from tissue of the chest wall and thoracopulmonary paraspinous musculature. Such a tumor arising from the kidney is quite rare, and few cases have been reported. The treatment is surgical removal and combined radiation and chemotherapy. The case described here is that of primitive neuroectodermal tumor arising from the kidney in a 25 -yearold woman who had abdominal pain and a mass. This case is a rare presentation of this tumor as an organ-based neoplasm.

\section{Case Report}

A previously healthy 25 -year-old woman came to the emergency department complaining of abdominal pain of 2 weeks' duration. The pain had worsened during the preceding 2 days. She had no fever, chills, nausea, vomiting, diarrhea, or change in bowel habits. The pain was described as constant, with no change related to eating. The patient described the pain as initially a sharp, stabbing pain, with some radiation to the back. As the course of the illness progressed, the pain became dull and constant. For the past 2 to 3 days before her visit, the pain has been constant with no relieving or exacerbating factors. This patient sought treatment because of worsening pain, progressive discomfort, and inability to obtain relief by such over-thecounter medications as antacids and acetaminophen. The patient had no history of any intraabdominal disease. She denied any hematuria, dysuria, pain, or burning on urination; bloody

Submitted, revised, 19 November 1999.

From the Emergency Medicine Section, Department of Surgery, University of Missouri, Columbia. Address reprint requests to Jack Wells, MD, Emergency Services, DC029.00, One Hospital Drive, Columbia, MO 65212. stools; or melenic stools. She denied any possibility of pregnancy.

Her medical history was notable for two abnormal Papanicolaou smear results within the last year; however, the patient stated that she had not had any follow-up care or evaluation. She admitted to smoking one-half pack of cigarettes per day ( 7.5 pack years) and admitted to alcohol intake of five to six beers a day. She denied use of recreational drugs. She had history of Legg-Calvé-Perthes disease as a teenager (bacterial disease caused by infection with Legionella pneumophila). She had no previous history of an operation.

On physical examination her blood pressure was $130 / 59 \mathrm{~mm} \mathrm{Hg}$, pulse was 92 beats per minute, respirations were $18 / \mathrm{min}$, and temperature was $37.3^{\circ} \mathrm{C}$. Findings on examination of her head, eyes, ears, nose and throat were unremarkable. Her neck was supple and without adenopathy. Her heart was regular and without murmurs, and a pulmonary examination showed equal breath sounds bilaterally. Her abdomen was soft. A mass in the left upper quadrant filled the entire left side of the abdomen and was palpable to the pelvic rim. It was fixed, tender, and without any associated peritoneal signs. Initially the mass was estimated to be approximately $10 \mathrm{~cm}$ long, extending from the left subcostal margin to the anterior superior iliac crest on the left side. The mass did not cross the midline, but extended laterally to the anterior axillary line. As the mass was palpated inferiorly, it became more difficult to follow, presumably because it became a deeper structure. There was no well-defined inferior border. Bowel sounds were normal. There were no inguinal nodes. Findings of a pelvic examination were normal, with no cervical discharge. Normal menstrual flow was observed. The uterus was not enlarged, and there were no adnexal masses or tenderness.

Her laboratory values were as follows: sodium $141 \mathrm{mEq} / \mathrm{L}$, potassium $3.9 \mathrm{mEq} /$, chloride 108 $\mathrm{mEq} / \mathrm{L}$, carbon dioxide content $24 \mathrm{mEq} / \mathrm{L}$, glucose $90 \mathrm{mg} / \mathrm{dL}$, blood urea nitrogen $10 \mathrm{mg} / \mathrm{dL}$, and 


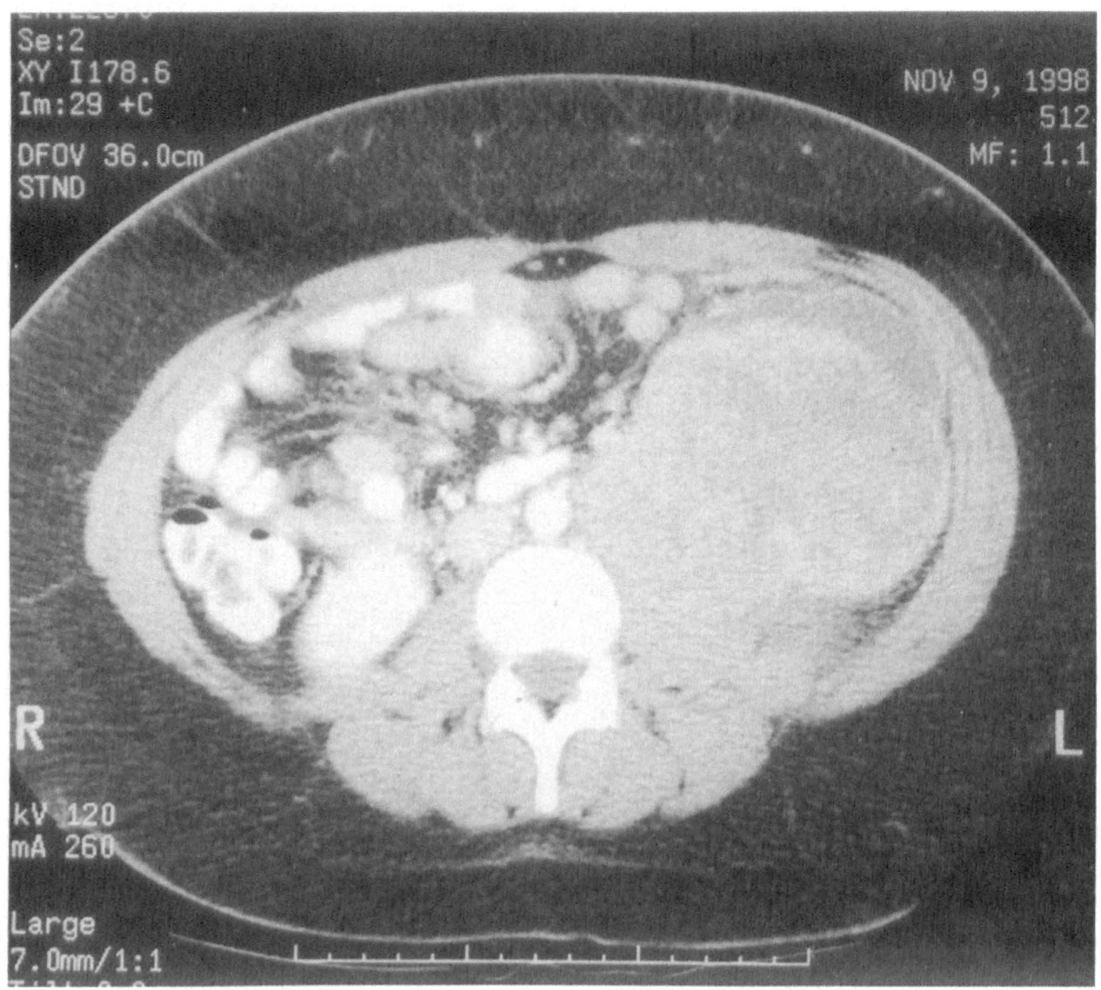

mor, because they are characteristic of this tumor. Accordingly, specific immunohistochemical staining was used to confirm the diagnosis. Results were positive using stains and techniques specific for neuroectodermal tumors, specifically cytokeratin and neuron-specific enolase. Stains for synaptophysin and vimentin, which also correlate highly with neuroectodermal tumor, showed weak reactivity. Electron microscopy showed poor ultrastructural preservation.

The diagnosis of primitive neuroectodermal tumor was made based on the strong positive correlation of the microscopic staining and immunohistochemical staining. Three consulting pathologists also came to the same diagnosis in light of the characteristic Homer-Wright rosette pattern and high correlation with confirmation by histochemical staining.

Tumor cells were also found in 2 of 23 lymph nodes submitted, including one para-aortic node. Radiation and chemotherapy were recommended, and further evaluation consisted of a bone scan, which was negative, and a postoperative CT scan of her chest, abdomen, and pelvis, which was negative. A bone marrow evaluation was negative for malignant cells. The patient started radiation and cheThis pattern and appearance on the initial evaluation raised the possibility of neuroectodermal tu- 


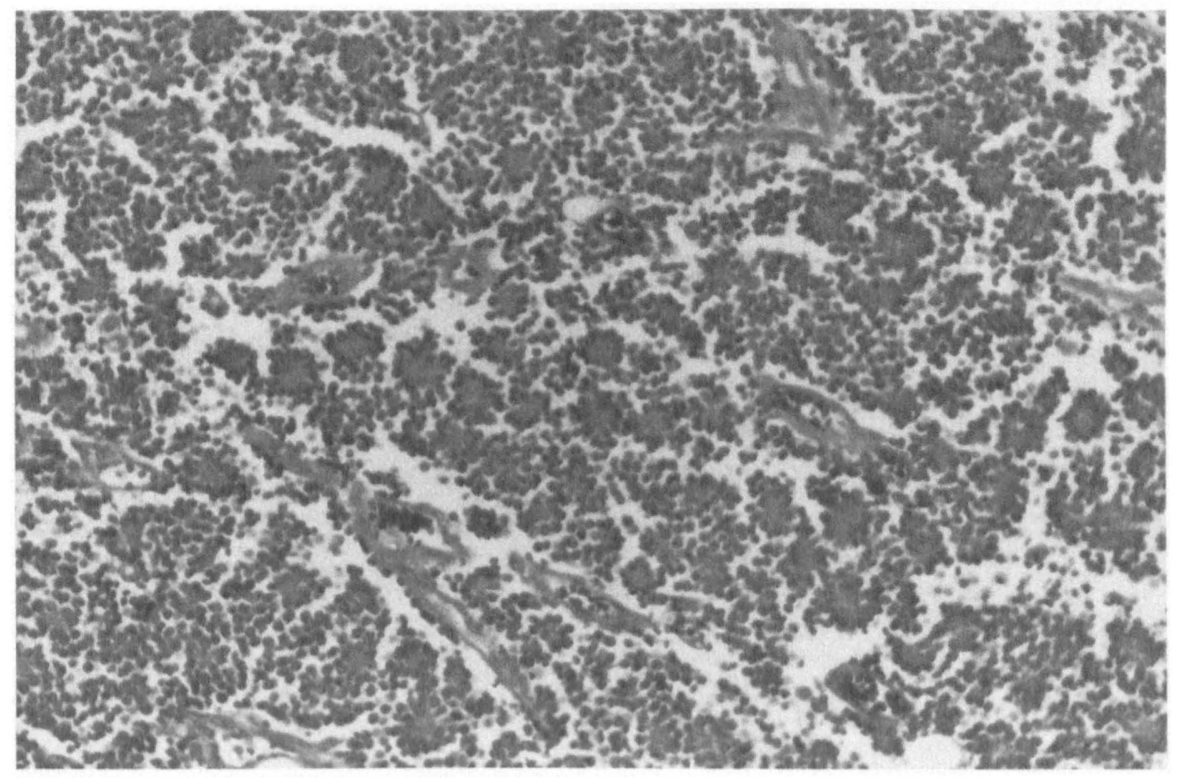

Figure 2. Microscopic section of the tumor showing Homer-Wright rosette formation.

motherapy and was discharged from the hospital. She has subsequently been readmitted to the hospital on two other occasions for complications related to her treatment, including radiation-induced colitis and profound granulocytopenia.

\section{Discussion}

Primitive neuroectodermal tumor has been classified as belonging to the Ewing family of sarcomas. $^{2-4}$ These tumors were first described by Stout in association with peripheral nerves. ${ }^{5}$ Children and adolescents are most frequently affected, with occurrences in the adult years rare. The most common locations are the head, neck, trunk, and extremities. ${ }^{5,6}$ These tumors have a poor prognosis, with a disease-free survival of $45 \%$ at 7.5 years. ${ }^{7}$

The differential diagnosis of renal tumors in this age-group includes renal cell tumor, Wilms tumor, and lymphoma. ${ }^{7,8}$ The diagnosis of primitive neuroectodermal tumor was made in view of the cytologic picture of the surgical specimen, the HomerWright rosette formations, and the high degree of correlation with immunohistochemical staining to confirm this particular tumor. The distinguishing genetic factor in primitive neuroectodermal tumor is the association with a translocation between chromosomes 11 and 22 , the $t(11 ; 22)(\mathrm{q} 24 ; \mathrm{q} 12) .^{7-10}$ Poorly differentiated rosettes are found on HomerWright staining. ${ }^{70,11}$ Immunohistochemical stain- ing for cytokeratin and neuron-specific enolase are usually positive, as are stains for vimentin and synaptophysin. ${ }^{11}$ All these markers point to a diagnosis of primitive neuroectodermal tumor. It is currently thought that the classification of primitive neuroectodermal tumor should be separate from the Ewing sarcoma, as the prognosis is worse for Ewing sarcoma because it is more invasive. ${ }^{10}$

The treatment of primitive neuroectodermal tumor is surgical resection and a chemotherapeutic regimen consisting of combinations of doxorubicin, cyclophosphamide, vincristine, and dactinomycin. In some cases chemotherapy is started before surgery. ${ }^{8}$ Radiation therapy is also used.

Described is a case of a rare tumor of which there have been few previously reported cases. ${ }^{1,7,10,11}$ The pathologic picture made a strong case for the diagnosis of primitive neuroectodermal tumor. A review of the literature indicates fewer than 10 previously reported cases of primitive neuroectodermal tumor. This diagnosis should be considered for primary kidney tumor in patients of this age-group. It is interesting to note that this patient had two Papanicolaou smears during the time immediately before the diagnosis of this tumor. It is likely that the tumor might have been palpable during those examinations. Often family physicians have the first opportunity to make the diagnosis of a rare disease by performing careful and thorough examinations during office visits. 


\section{References}

1. Seaff M, McManus A, Scheimberg I, Paris A, Shipley J, Baithun S. Primitive neuroectodermal tumor of the kidney confirmed by fluorescence in situ hybridization. Am J Surg Pathol 1997;4:461-8.

2. Verrill MW, Judson IR, Harmer CL, Fisher C, Thomas JM, Wiltshaw E. Ewing's sarcoma and primitive neuroectodermal tumor in adults. are they different from Ewing's sarcoma and primitive neuroectodermal tumor in children? J Clin Oncol 1997; 15:2611-21.

3. Antoneli CB, Costa CM, de Camargo B, Sredni ST, Alfer $\mathrm{W}$ Jr., Chojniak R. Primitive neuroectodermal tumor (PNET)/extraosseous Ewing sarcoma of the kidney. Med Pediatr Oncol 1998;30:303-7.

4. Benesch $M$, Urban $C$. Is primitive neuroectodermal tumor of the kidney a distinct entity? Cancer 1998; 82:1414-6.

5. Hollis LJ, Poole S, Hern J, Patel KS. Primitive neuroectodermal tumor of the masseter muscle. J Laryngol Otol 1996;110:1179-81.

6. Akeyson EW, McCutcheon IE, Pershouse MA,
Steck PA, Fuller GN. Primitive neuroectodermal tumor of the median nerve. Case report with cytogenic analysis. J Neurosurg 1996;85:163-9.

7. Furman J, Murphy WM, Jelsma PF, Garzotto MG, Marsh RD. Primary primitive neuroectodermal tumor of the kidney. Case report and review of the literature. Am J Clin Pathol 1996;106:339-44.

8. Patel SR, Benjamin RS. Sarcomas of soft tissue and bone. In: Fauci AS, Harrison TR, editors. Harrison's principles of internal medicine. 14th ed. New York: McGraw-Hill, 1998.

9. McManus AP, Gusterson BA, Pinkerton CR, Shipley JM. The molecular pathology of small round-cell tumors-relevance to diagnosis, prognosis, and classification. J Pathol 1996;178:116-21.

10. Dehner LP, Primitive neuroectodermal tumor and Ewing's sarcoma. Am J Surg Pathol 1993;17:1-13.

11. Marley EF, Liapis H, Humphrey PA, et al. Primitive neuroectodermal tumor of the kidney - another enigma: a pathologic, immunohistochemical, and molecular diagnostic study. Am J Surg Pathol 1997; 21:354-9. 